\title{
Are physical activity interventions in primary care and the community cost-effective? A systematic review of the evidence
}

\author{
Sue Garrett, C Raina Elley, Sally B Rose, Des O’Dea, Beverley A Lawton and Anthony C Dowell
}

\begin{abstract}
Background

The health and economic burden of physical inactivity is well documented. A wide range of primary care and community-based interventions are available to increase physical activity. It is important to identify which components of these interventions provide the best value for money.

Aim

To assess the cost-effectiveness of physical activity interventions in primary care and the community.

Design of study

Systematic review of cost-effectiveness studies based on randomised controlled trials of interventions to increase adult physical activity that were based in primary health care or the community, completed between 2002 and 2009

\section{Method}

Electronic databases were searched to identify relevant literature. Results and study quality were assessed by two researchers, using Drummond's checklist for economic evaluations. Cost-effectiveness ratios for moving one person from inactive to active, and costutility ratios (cost per quality-adjusted life-year [QALY]) were compared between interventions.

Results

Thirteen studies fulfilled the inclusion criteria. Eight studies were of good or excellent quality. Interventions, study populations, and study designs were heterogeneous, making comparisons difficult. The cost to move one person to the 'active' category at 12 months was estimated for four interventions ranging from $€ 331$ to $€ 3673$. The cost-utility was estimated in nine studies, and varied from $€ 348$ to $€ 86877$ per QALY.

\section{Conclusion}

Most interventions to increase physical activity were cost-effective, especially where direct supervision or instruction was not required. Walking, exercise groups, or brief exercise advice on prescription delivered in person, or by phone or mail appeared to be more costeffective than supervised gym-based exercise classes or instructor-led walking programmes. Many physical activity interventions had similar cost-utility estimates to funded pharmaceutical interventions and should be considered for funding at a similar level.

Keywords

costs and cost analysis; exercise; primary health care; review, systematic.
\end{abstract}

\section{INTRODUCTION}

The prevalence of physical inactivity remains high in developed and developing countries. ${ }^{1}$ Not only does physical inactivity contribute to increased prevalence of chronic conditions such as cardiovascular disease, obesity, type 2 diabetes, osteoporosis, colon cancers, depression, and fall-related injuries, but it also contributes to between $1.5 \%$ and $3.0 \%$ of direct healthcare costs in developed countries. ${ }^{1} \mathrm{~A}$ wide range of interventions have been shown to increase physical activity. ${ }^{2}$ However, it is essential to identify which components provide the best value for money.

Physical activity counselling in primary health care has been recommended. ${ }^{3}$ In some countries at least $80 \%$ of the population visit primary health care annually, ${ }^{4,5}$ making this an ideal setting for intervening to increase physical activity. Furthermore, patients expect to receive health-related messages in this

$S$ Garrett, MPH, Research fellow; SB Rose, PhD, senior research fellow; BA Lawton, ONZM, FRNZCGP, DObst, director, Women's Health Research Centre; AC Dowell, FRCGP, FRNZCGP, professor, Department of Primary Health Care and General Practice; D O'Dea, BSc, BA, lecturer, Department of Public Health, University of Otago, Wellington, New Zealand.CR Elley, PhD, MBChB, senior lecturer, Department of General Practice and Primary Health Care, School of Population Health, University of Auckland, Auckland, New Zealand.

Address for correspondence

Sue Garrett, Women's Health Research Centre, Department of Primary Health Care and General Practice, University of Otago, Wellington, PO Box 7343, Wellington South, New Zealand. E-mail: susan.garrett@otago.ac.nz

Submitted: 23 March 2010; Editor's response: 24 May 2010; final acceptance: 27 July 2010.

(0)British Journal of General Practice

This is the full-length article (published online 28 Feb 2011) of an abridged version published in print. Cite this article as: Br J Gen Pract 2011; DOI: 10.3399/bjgp11X561249. 
context and may therefore be more receptive to brief advice or referral to community-based interventions. It is still not known if physical activity interventions in this context are cost-effective, or which types of intervention are the most cost-effective. Nor is it known how cost-effective these interventions are when compared with other interventions in primary health care, such as pharmaceutical interventions that are also aimed at reducing chronic disease.

Physical activity interventions based in primary health care, such as exercise on prescription, have been shown to be effective ${ }^{6-9}$ and cost-effective, ${ }^{10,11}$ with a cost-utility ratio comparable to many currently-funded pharmaceutical therapies. A systematic review of the cost-effectiveness of physical activity interventions within primary health care was completed in 2002, in which eight studies were identified, published between 1996 and 2002. ${ }^{12}$ The review found that there was a trend towards favourable cost-effectiveness of physical activity promotion through primary health care but there were few trials available of mixed study quality. The cost-effectiveness of community-based physical activity promotion in general was assessed by another systematic review which also showed favourable results, but included modelling studies and evaluations of workplace or infrastructural innovations in the community, as well as individualbased interventions to promote physical activity. ${ }^{13}$

The current systematic review assesses the evidence for cost-effectiveness of physical activity interventions in the community, particularly in primary care, and only those based on randomised controlled trials conducted since the previous review of primary care evidence in $2002 .^{12}$

\section{METHOD}

\section{Search strategy}

A literature search was carried out using eight healthrelated electronic databases (Table 1).

\section{How this fits in}

Community- and primary healthcare-based physical activity interventions have been shown to be effective in increasing population physical activity levels. The cost-effectiveness of these interventions varies and it is still not known if physical activity interventions in this context are cost-effective, which types of

intervention are the most cost-effective, and how these interventions compare with others delivered in primary health care, such as pharmaceutical interventions. This study found that many interventions to increase physical activity were within the generally accepted range of cost-effectiveness, especially where direct supervision or instruction was not required. Based on the higher-quality studies, it is possible to deliver a physical activity intervention for between $€ 1120$ and $€ 15860$ per quality-adjusted life-year gained, which is more cost-effective than many other currently-funded pharmaceutical interventions.

\section{Selection process}

To be included in the systematic review, studies had to be economic analyses of lifestyle interventions that included physical activity advice and/or programmes for adults, and were based in either primary care or the community, published in English since 2002, used general study populations or those with disease states known to be improved by physical activity, and had a follow-up period of at least 6 months. Only those cost analyses that had been conducted in association with randomised controlled trials of physical activity interventions were included. The review excluded studies that were based in the workplace, used economic modelling rather than actual costs, were economic analyses based on case-studies, surveys, non-experimental studies, or theoretical scenarios, involved unique disease-state populations (for example cardiac rehabilitation patients), or did not include either cost per physical activity measure or cost per quality-adjusted life-year (QALY) as outcomes.

\section{Data extraction and analysis}

Data extraction was undertaken by the primary author and verified by a second author. Type of

Table 1. Literature search strategy of electronic databases, and subject headings used to identify cost-effectiveness studies.

\begin{tabular}{llll} 
Databases & $\begin{array}{l}\text { Physical activity } \\
\text { subject headings }\end{array}$ & $\begin{array}{l}\text { Cost-effectiveness } \\
\text { headings }\end{array}$ & $\begin{array}{l}\text { Healthcare setting } \\
\text { subject headings }\end{array}$ \\
\hline $\begin{array}{l}\text { MEDLINE } \\
\text { Emb-present })\end{array}$ & Exercise & Economics & Primary health care \\
\hline PsycINFO & Physical activity & Health economics & General practice \\
\hline SPORTDiscus & Fitness & Cost-minimisation analysis & Family practice \\
\hline CINAHL & Exertion & Cost-benefit analysis & Primary medical care \\
\hline Cochrane Database of & & Cost-effectiveness analysis & Family medicine \\
Systematic Reviews services & & Cost-utility analysis & Community health \\
\hline Web of Knowledge & & Healthcare costs & \\
\hline Scopus & & Costs and cost analysis &
\end{tabular}


Table 2. Critical appraisal of selected studies.

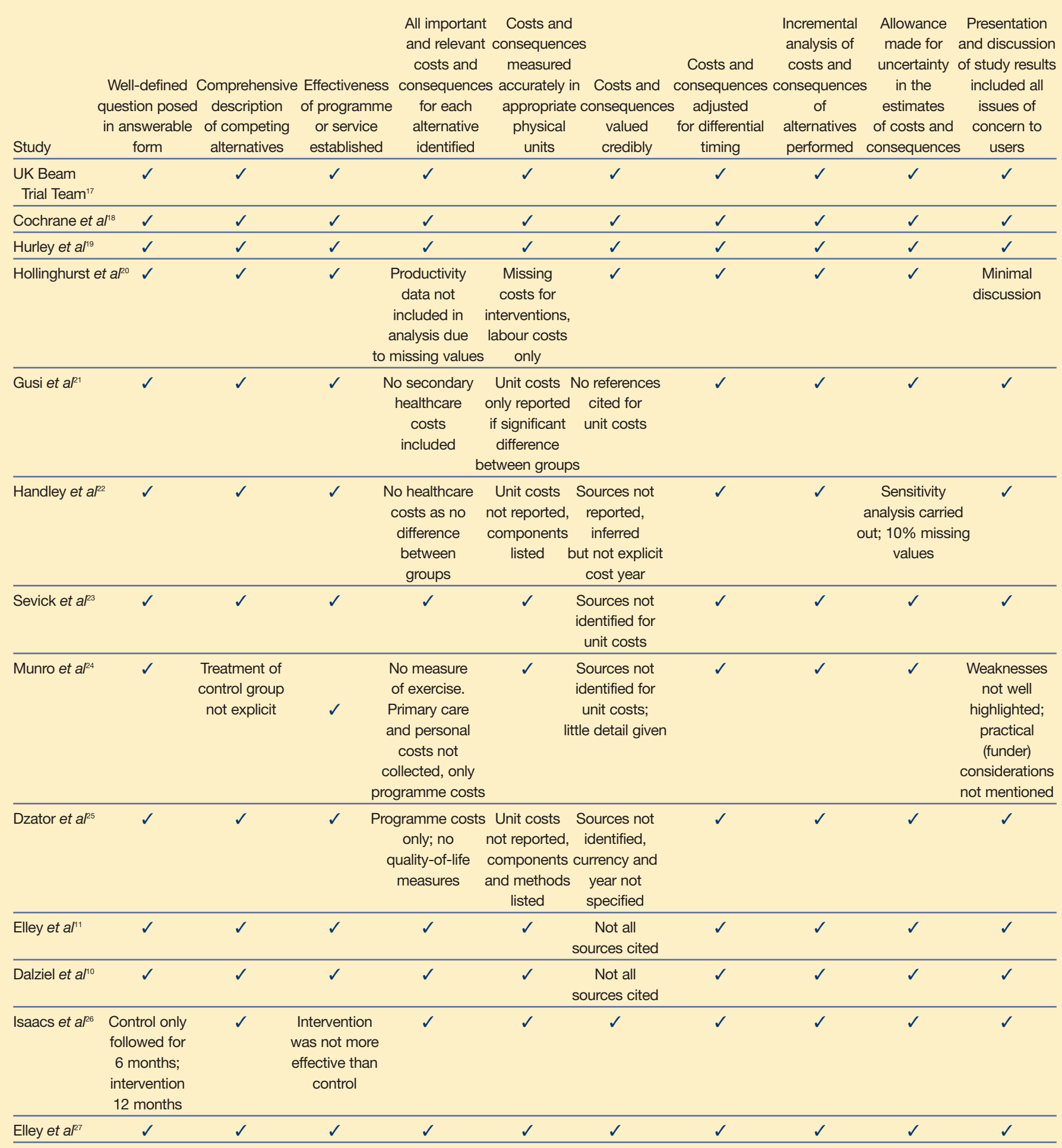

economic analysis and perspective, intervention and comparison, participants, follow-up duration, and outcome were recorded from each study that fulfilled the inclusion criteria. Studies were assessed for methodological quality using Drummond's checklist for assessing economic evaluations (Table 2). ${ }^{14}$
Based on the extent to which each study met Drummond's criteria, a rating of 'poor', 'fair', 'good', or 'excellent' was assigned and is listed in the first column of Table 3.

To compare the economic results of individual studies, all costs were converted to Euros (€), ${ }^{15}$ using 
Table 3. Cost-effectiveness studies undertaken of interventions that included physical activity counselling or intervention within primary care or the community (published from 2002 to 2009).

\begin{tabular}{|c|c|c|c|c|c|c|c|c|}
\hline \multirow[b]{2}{*}{$\begin{array}{l}\text { Study details } \\
\text { and quality }\end{array}$} & \multirow[b]{2}{*}{$\begin{array}{l}\text { Objective; } \\
\text { economic } \\
\text { perspective }\end{array}$} & \multirow[b]{2}{*}{$\begin{array}{l}\text { Study type; } \\
\text { economic } \\
\text { analysis type }\end{array}$} & \multirow[b]{2}{*}{$\begin{array}{l}\text { Interventions (l) } \\
\text { and } \\
\text { comparison (C) }\end{array}$} & \multirow[b]{2}{*}{$\begin{array}{l}\text { Participants, } \\
\text { number in } \\
\text { each group }\end{array}$} & \multirow[b]{2}{*}{$\begin{array}{l}\text { Follow-up } \\
\text { duration } \\
\text { (months) }\end{array}$} & \multicolumn{3}{|c|}{ Outcome } \\
\hline & & & & & & $\begin{array}{l}\text { Annual cost per } \\
\text { participant to } \\
\text { become active, } \\
\text { (€at time of } \\
\text { study) [inflated } \\
\text { to June 2008] }\end{array}$ & $\begin{array}{l}\text { Cost of } \\
\text { shifting to active } \\
\text { category }{ }^{2},(€ \text { at } \\
\text { time of study) } \\
\text { [inflated to } \\
\text { June 2008] }\end{array}$ & $\begin{array}{l}\text { Cost per } \\
\text { QALY (€ } \\
\text { at time of } \\
\text { study) } \\
\text { [inflated to } \\
\text { June 2008] }\end{array}$ \\
\hline $\begin{array}{l}\text { UK Beam } \\
\text { Trial Team, }{ }^{17} \\
\text { UK: } \\
\text { Excellent }\end{array}$ & $\begin{array}{l}\text { Primary care, } \\
\text { multifaceted } \\
\text { intervention } \\
\text { for low back } \\
\text { pain; health } \\
\text { funder, } \\
2000-2001\end{array}$ & RCT; CUA & $\begin{array}{l}\text { I1: exercise } \\
\text { programme; } \\
\text { I2: spinal } \\
\text { manipulation; } \\
\text { I3: combined; } \\
\text { C: usual care }\end{array}$ & $\begin{array}{l}\text { Participants } \\
\text { consulting GP } \\
\text { for low back } \\
\text { pain: I1: } 297 \\
\text { I2: } 342 \\
\text { I3: } 322 \\
\text { C: } 326\end{array}$ & 12 & & & $\begin{array}{l}£ 8235^{\mathrm{b}} \\
(€ 13423) \\
{[€ 15860]}\end{array}$ \\
\hline $\begin{array}{l}\text { Cochrane et } a l,{ }^{18} \\
\text { UK: } \\
\text { Excellent }\end{array}$ & $\begin{array}{l}\text { Primary care/ } \\
\text { community } \\
\text { water exercise } \\
\text { programme; } \\
\text { societal costs, } \\
2002\end{array}$ & RCT; CEA & $\begin{array}{l}\text { 11: water-based } \\
\text { exercise, } \\
\text { C: usual care }\end{array}$ & $\begin{array}{l}\text { Older } \\
\text { participants } \\
\text { with hip and/ } \\
\text { or knee OA: } \\
\text { I1: } 153 \\
\text { C: } 159\end{array}$ & 12 & & & $\begin{array}{l}£ 5008^{\circ} \\
(€ \boxminus 963) \\
{[€ 9160]}\end{array}$ \\
\hline $\begin{array}{l}\text { Hurley et } a /{ }^{19} \\
\text { UK: } \\
\text { Excellent }\end{array}$ & $\begin{array}{l}\text { Primary care, } \\
\text { knee } \\
\text { rehabilitation; } \\
\text { societal costs, } \\
2003\end{array}$ & $\begin{array}{l}\text { Cluster RCT; } \\
\text { CEA, CUA }\end{array}$ & $\begin{array}{l}\text { I1: individual } \\
\text { rehabilitation, }{ }^{\mathrm{d}} \\
\text { I2: group-based } \\
\text { rehabilitation, } \\
\text { C: usual primary } \\
\text { care }\end{array}$ & $\begin{array}{l}\text { Participants } \\
\geq 50 \text { years } \\
\text { attending primary } \\
\text { care for mild/ } \\
\text { moderate/ } \\
\text { severe knee } \\
\text { pain, duration } \\
>6 \text { months: } \\
\text { I1: } 146 \\
\text { I2: } 132 \\
\text { C: } 140\end{array}$ & 6 & & & $\begin{array}{l}\text { Usual care } \\
\text { produced } \\
\text { greater QALY } \\
\text { gain }(0.0096) \\
\text { than individual } \\
\text { rehabilitation } \\
(-0.0034) \text { and } \\
\text { group } \\
\text { rehabilitation } \\
(0.0057) \text {, despite } \\
\text { 'functional } \\
\text { improvements' } \\
\text { with exercise } \\
\text { interventions }\end{array}$ \\
\hline $\begin{array}{l}\text { Hollinghurst } \\
\text { et al, },^{20} \mathrm{UK} \text { : } \\
\text { Fair }\end{array}$ & $\begin{array}{l}\text { Primary care; } \\
\text { societal } \\
\text { perspective, } \\
2005\end{array}$ & $\begin{array}{l}4 \times 2 \text { factorial } \\
\mathrm{RCT} \text {; CEA }\end{array}$ & $\begin{array}{l}\text { 11: short-course } \\
\text { Alexander } \\
\text { technique, I2: } \\
\text { long-course } \\
\text { Alexander } \\
\text { technique, } \\
\text { I3: massage, } \\
\text { C: usual care } \\
\text { groups; All also } \\
\text { randomised into } \\
\text { with or without GP } \\
\text { exercise script anc } \\
\text { practice nurse } \\
\text { exercise counsellir }\end{array}$ & $\begin{array}{l}\text { Participants } \\
\text { with chronic or } \\
\text { recurrent low } \\
\text { back pain: } \\
\text { I1: } 144 \\
\text { I2: } 144 \\
\text { I3: } 147 \\
\text { C: } 144 \\
\text { P } \\
\text { ling }\end{array}$ & 12 & & & $\begin{array}{l}£ 2847 \\
(€ 4157) \\
\text { [€4577] } \\
\text { (exercise } \\
\text { counselling and } \\
\text { prescription } \\
\text { for exercise } \\
\text { compared with } \\
\text { usual care) }\end{array}$ \\
\hline $\begin{array}{l}\text { Gusi et al, }{ }^{21} \\
\text { Spain: } \\
\text { Poor }\end{array}$ & $\begin{array}{l}\text { Primary care, } \\
\text { supervised } \\
\text { walking } \\
\text { programme; } \\
\text { health funder, } \\
2005\end{array}$ & RCT; CUA & $\begin{array}{l}\text { I1: walking- } \\
\text { based, } \\
\text { supervised } \\
\text { programme } \\
(3 \times 50 \\
\text { min/week), } \\
\text { C: usual care }\end{array}$ & $\begin{array}{l}\text { Women, } \\
\geq 60 \text { years with } \\
\text { moderate } \\
\text { depression or } \\
\text { overweight: } \\
\text { I1: } 64 \\
\text { C: } 63\end{array}$ & 6 & & & $€ 311$ [€348] \\
\hline
\end{tabular}

annual average exchange rates. This method has been used previously. ${ }^{13}$ Costs have then been converted to the reference quarter of June 2008 using consumer price index (CPI) adjustments from each country and are presented as such throughout. ${ }^{16}$ Indices of consumer prices were used in preference to national indices of healthcare costs, because of the accessibility and international comparability of the 
Table 3 continued. Cost-effectiveness studies undertaken of interventions that included physical activity counselling or intervention within primary care or the community (published from 2002 to 2009).

\begin{tabular}{|c|c|c|c|c|c|c|c|c|}
\hline $\begin{array}{l}\text { Handley et al, }{ }^{22} \\
\text { US: } \\
\text { Fair }\end{array}$ & $\begin{array}{l}\text { Primary care- } \\
\text { based automated } \\
\text { telephone } \\
\text { support; direct } \\
\text { programme } \\
\text { costs, June } \\
2003 \text { to } \\
\text { December } 2004\end{array}$ & $\begin{array}{l}\text { RCT; CEA, } \\
\text { CUA }\end{array}$ & $\begin{array}{l}\text { I1: automated } \\
\text { telephone } \\
\text { surveillance/ } \\
\text { support, nurse } \\
\text { care } \\
\text { management, } \\
\text { C: usual care }\end{array}$ & $\begin{array}{l}\text { Adult English-, } \\
\text { Spanish-, } \\
\text { Cantonese- } \\
\text { speaking primary } \\
\text { care participants } \\
\text { with type } 2 \\
\text { diabetes: I1: } 112 \\
\text { C: } 114\end{array}$ & 12 & $\begin{array}{l}\text { US \$558 } \\
(€ 463)[€ 551]\end{array}$ & & $\begin{array}{l}\text { US \$65 } 167 \\
\text { (€54 089), } \\
{[€ 64346]}\end{array}$ \\
\hline $\begin{array}{l}\text { Sevick et al, }{ }^{23} \\
\text { US: } \\
\text { Excellent }\end{array}$ & $\begin{array}{l}\text { Community, } \\
\text { physical activity } \\
\text { counselling; } \\
\text { health funder, } \\
2004\end{array}$ & RCT; CEA & $\begin{array}{l}\text { I1: telephone- } \\
\text { based feedback } \\
\text { on PA', I2: print- } \\
\text { based feedback } \\
\text { on PA, C: contact } \\
\text { control }\end{array}$ & $\begin{array}{l}\text { Sedentary adults } \\
\text { (18-65 years): } \\
\text { I1: } 80 \\
\text { I2: } 81 \\
\text { C: } 78\end{array}$ & 12 & & $\begin{array}{l}\$ 3967 \text { (€3174) } \\
{[€ 3673]} \\
\text { (phone group); } \\
\$ 955 \text { (€764) } \\
{[€ 884]} \\
\text { (print group) }\end{array}$ & \\
\hline $\begin{array}{l}\text { Munro et al, }{ }^{24} \\
\text { UK: } \\
\text { Poor }\end{array}$ & $\begin{array}{l}\text { Primary care, } \\
\text { exercise class; } \\
\text { health funder, } \\
2003 / 2004\end{array}$ & $\begin{array}{l}\text { Cluster RCT; } \\
\text { CUA, CEA }\end{array}$ & $\begin{array}{l}\text { I1: free, } 2 \text { x } \\
\text { weekly ( } 45 \text { min) } \\
\text { community } \\
\text {-based exercise } \\
\text { class, } \\
\text { C: usual care }\end{array}$ & $\begin{array}{l}\text { Participants } \\
\geq 65 \text { years } \\
\text { assessed by surve } \\
\text { as being in least- } \\
\text { active four-fifths } \\
\text { of the population: } \\
\text { I1: } 2283 \\
\text { C: } 4137\end{array}$ & 24 & & & $\begin{array}{l}€ 17174 \\
{[€ 19425]}\end{array}$ \\
\hline $\begin{array}{l}\text { Dzator et al, }{ }^{25} \\
\text { Australia: } \\
\text { Fair }\end{array}$ & $\begin{array}{l}\text { Physical activity/ } \\
\text { nutrition } \\
\text { programme in } \\
\text { community } \\
\text { setting; direct } \\
\text { programme costs }\end{array}$ & RCT; CEA & $\begin{array}{l}\text { I1: high-level, } \\
\text { interactive group } \\
\text { sessions, I2: } \\
\text { low-level, mailed } \\
\text { intervention, } \\
\text { C: no intervention }\end{array}$ & 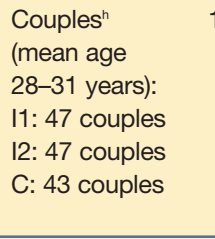 & 12 & $\begin{array}{l}\text { No significant } \\
\text { effect difference } \\
\text { between groups } \\
\text { observed regardin } \\
\text { the activity } \\
\text { level: exercise } \\
\text { days per week }\end{array}$ & & $\begin{array}{l}\text { AUS \$460.44 } \\
\text { (€267) [€350] } \\
\text { (high level); } \\
\text { AUS \$458.61 } \\
\text { (€266) [€349] } \\
\text { (low level) }\end{array}$ \\
\hline $\begin{array}{l}\text { Elley et } a{ }^{11} \\
\text { New Zealand: } \\
\text { Excellent }\end{array}$ & $\begin{array}{l}\text { Primary care } \\
\text { exercise } \\
\text { counselling/ } \\
\text { prescription; } \\
\text { societal costs, } \\
2001\end{array}$ & $\begin{array}{l}\text { Cluster RCT; } \\
\text { CEA }\end{array}$ & $\begin{array}{l}\text { I1: Green } \\
\text { Prescription, } \\
\text { counselling in } \\
\text { general practice, } \\
\text { C: usual care }\end{array}$ & $\begin{array}{l}\text { Less active } \\
\text { primary care } \\
\text { participants } \\
\text { (40-79 years): } \\
\text { I1: } 451 \\
\text { C: } 427\end{array}$ & 12 & $\begin{array}{l}€ 825 \text { [€957] } \\
\text { (activity level } 5 \times \\
30 \mathrm{~min} / \text { week) }\end{array}$ & $\begin{array}{l}\text { NZ \$17569 } \\
(€ 825)[€ 957]\end{array}$ & \\
\hline $\begin{array}{l}\text { Isaacs et al, }{ }^{26} \\
\text { UK } \\
\text { Good }\end{array}$ & $\begin{array}{l}\text { Primary care } \\
\text { referral to exercise } \\
\text { programmes; } \\
\text { health funder, } \\
2002 \text { costs }\end{array}$ & RCT; CEA & $\begin{array}{l}\text { I1: supervised, } \\
\text { gym-based } \\
\text { exercise } \\
\text { classes,' I2: } \\
\text { instructor-led } \\
\text { walking } \\
\text { programme,' } \\
\text { C: advice and } \\
\text { information only }\end{array}$ & $\begin{array}{l}\text { Physically } \\
\text { inactive } \\
40-74 \text { year olds } \\
\text { with at least one } \\
\text { cardiovascular } \\
\text { risk factor: } \\
\text { I1: } 317 \\
\text { I2: } 311 \\
\text { C: } 315\end{array}$ & 12 & & & $\begin{array}{l}\text { I1: } £ 19500 \\
(€ 31005) \\
{[€ 35665] ;} \\
\text { I2: } £ 47500 \\
(€ 75525) \\
{[€ 86877]}\end{array}$ \\
\hline $\begin{array}{l}\text { Elley et al, }{ }^{27} \\
\text { New Zealand: } \\
\text { Excellent }\end{array}$ & $\begin{array}{l}\text { Primary care } \\
\text { exercise } \\
\text { counselling/ } \\
\text { prescription; } \\
\text { societal costs, } \\
2008\end{array}$ & RCT; CEA & $\begin{array}{l}\text { I1: enhanced } \\
\text { Green } \\
\text { Prescription, } \\
\text { counselling in } \\
\text { primary care, } \\
\text { C: usual care }\end{array}$ & $\begin{array}{l}\text { Physically } \\
\text { inactive } \\
40-74 \text {-year-old } \\
\text { women }\end{array}$ & 24 & \multicolumn{3}{|c|}{$\begin{array}{l}\text { NZ \$687 [ } € 331] \\
\text { sustained at } \\
12 \text { months; NZ \$1407 } \\
\text { [€678] sustained } \\
\text { at } 24 \text { months }\end{array}$} \\
\hline
\end{tabular}

a Undertaking at least 150 minutes of at least moderate-intensity physical activity per week. ${ }^{b}$ Exercise programme component only. ${ }^{c} 20 \%$ ( $\left.\mathrm{n}=65\right)$ missing values for EuroQol-5D (EQ-5D) imputed by regression based on age, sex, and EuroQol-Visual Analogue Scale (EQ-VAS). ${ }^{\circ} 12$ supervised sessions, $2 \times$ weekly for 6 weeks (40 minutes to 1 hour) including information giving and exercises. ${ }^{\circ}$ Cost to achieve a $10 \%$ increase in the proportion of participants achieving moderate or vigorous physical activity. 'No significant difference between phone and control group at 12 months for PA measures. ${ }^{9}$ Programme cost of shifting one person from sedentary to active category. 'Variable proportion of participants reported as sufficiently active at baseline. 'Ten-week (2-3 times per week). CEA = cost-effectiveness analysis. CUA = cost-utility analysis. $O A=$ osteoarthritis. $P A=$ physical activity. $Q A L Y=$ quality-adjusted life-year. $R C T=$ randomised controlled trial. 
former. The cost of shifting one participant into the 'active' category (achieving 2.5 hours per week of at least moderate-intensity physical activity), the annual cost per participant to become 'active', and the cost per QALY gained are reported where available. The methods used for calculating changes in QALYs are provided in the source papers.

\section{RESULTS}

\section{Studies identified}

The literature search identified 696 publications, from which 38 were assessed in detail. Of those, 13 studies met the selection criteria and were included in this review (Figure 1). Studies were excluded for the following reasons: eight studies did not use outcome measures that were either cost per QALY gained or a physical activity measure; two studies targeted a very specific population for secondary prevention (for example, cardiac rehabilitation patients); two studies were set in the workplace; four studies included a combined intervention (physical activity plus a nutrition intervention, or physical activity plus a psychological intervention) where the impact of the physical activity intervention could not be isolated; and four analyses were based on inadequate trials, due to short follow-up (3 months), high attrition (45\%), insufficient power due to small sample size ( $n$ = 36), or absence of a comparison group.

The 13 studies that met the inclusion criteria are described in Table 3 and grouped by targeted population or condition (musculoskeletal conditions, obesity or depression, sedentary adults). The descriptions of the interventions, follow-up and incremental cost-effectiveness ratios for instructor-led and supervised exercise, exercise and nutrition

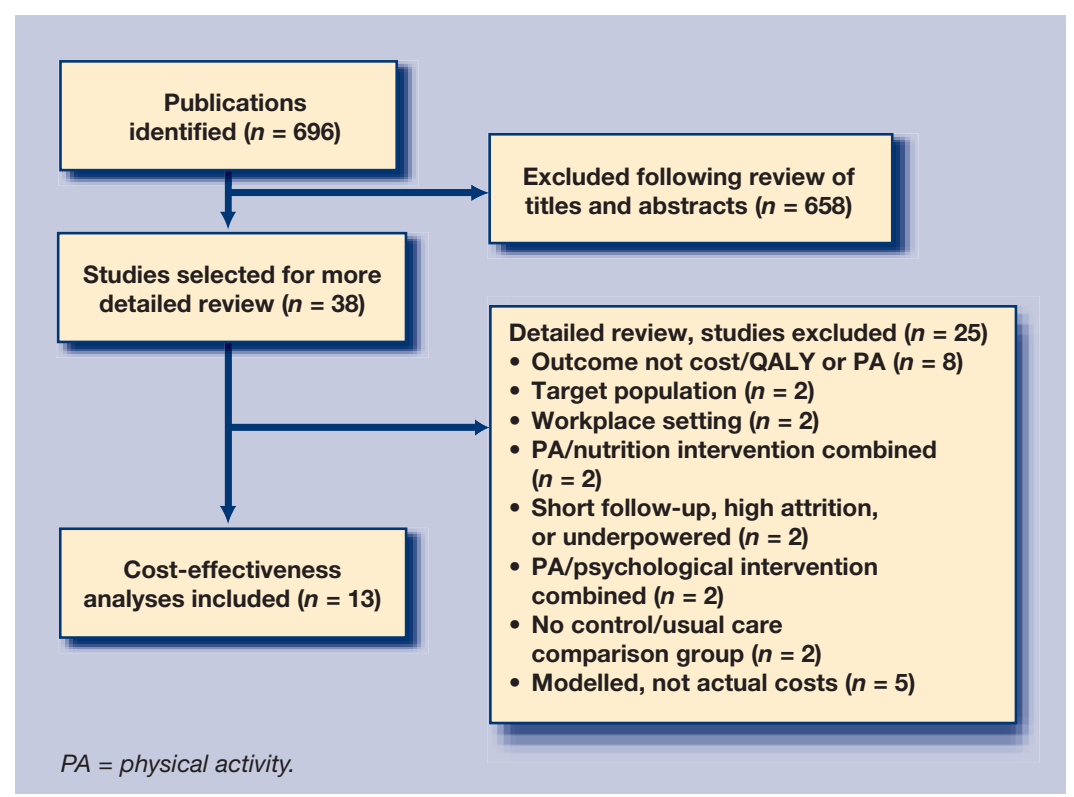

programmes, community walking, and brief counselling with exercise on prescription are also presented in Table 3. Community walking, exercise and nutrition programmes, and brief counselling with exercise on prescription (Green Prescription) had more favourable cost-effectiveness ratios than instructorled or supervised exercise sessions (Figure 2).

\section{DISCUSSION}

\section{Summary of main findings}

Most interventions to increase physical activity were cost-effective when compared with international thresholds for acceptable value for funded interventions,,$^{28}$ especially where direct supervision or instruction were not required. Walking, exercise
Figure 1. Flowchart of study selection.

Figure 2. Cost-utility (cost per QALY) for different physical activity interventions $(2008$ equivalent $€$ ).

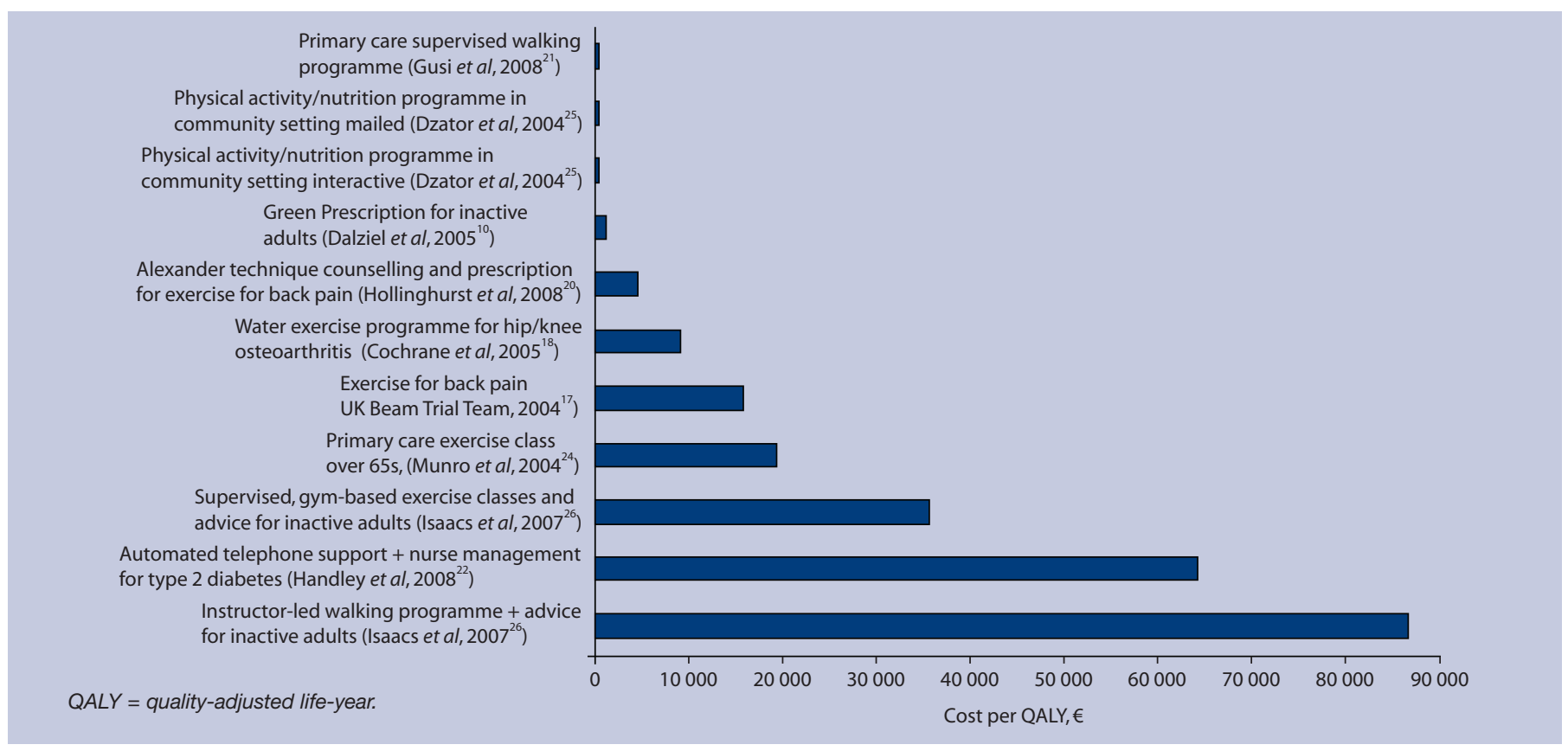


groups, or brief exercise advice on prescription delivered in person, or by phone or mail, had a lower cost per QALY compared with supervised gym-based exercise classes or instructor-led walking programmes. Many physical activity interventions had similar estimates of cost-utility to funded pharmaceutical interventions.

Costs per QALY gained varied substantially between the studies. The cost of moving one person from 'inactive' to 'active' at 12 months was estimated for four interventions, and ranged from $€ 331$ to $€ 3673$. Cost-utility was estimated in nine studies and varied from $€ 348$ to $€ 86877$ per QALY. Community walking, ${ }^{21}$ exercise and nutrition programmes, ${ }^{25}$ and brief advice with exercise on prescription (Green Prescription) $)^{10}$ were the most cost-effective with respect to cost-utility. The Green Prescription, ${ }^{6}$ enhanced Green Prescription, ${ }^{27}$ and printed material or phone-delivered advice ${ }^{23}$ had similar costeffectiveness ratios for moving one sedentary person to an active state.

When considering interventions for specific disease-based populations, the exercise prescription given by the GP and exercise counselling by the practice nurse for people with chronic or recurrent low back pain ${ }^{20}$ was more cost-effective ( $€ 4577$ per QALY) than the water-based exercise intervention for older participants with osteoarthritis of the hip and/or knee ( $€ 160$ per QALY)..$^{18}$ A weekly exercise class for sedentary people aged 65 years and older ${ }^{24}$ was more cost-effective ( $€ 19425$ per QALY) than both the supervised gym-based exercise classes and the instructor-led walking programme for sedentary 40-74 year olds ( $€ 35665$ and $€ 86877$ per QALY). ${ }^{26}$ The findings suggest that advice interventions, such as exercise on prescription and some group-based exercise programmes, are more cost-effective than individualised gym-based or instructor-led walking groups.

There is no universal threshold of cost per QALY gained to determine whether an intervention should be funded. However, most of the cost per QALY values from studies reviewed here were below the threshold reported by the National Institute for Health and Clinical Excellence (NICE) (£20 000-30 000), ${ }^{28}$ and lower than values reported for many funded pharmaceutical and other interventions for conditions such as diabetes (cholesterol control: $€ 58882$ per QALY gained; intensive glucose control: $€ 32610$ per QALY gained; case management: $€ 41452$ per QALY gained). ${ }^{29-32}$

It should be noted that one study found exercise interventions were effective for functional improvements compared with 'usual care'. ${ }^{19}$ However, greater gains in quality-of-life measures were found in the 'usual care' group than in the 'exercise' intervention groups (negative cost per QALY), so the cost per QALY was difficult to interpret. ${ }^{19}$

Comparing the annual cost per participant to become active is difficult due to the different definitions and analytical approaches used. Handley et al used the cost to achieve a $10 \%$ increase in the proportion of participants achieving recommended moderate or vigorous physical activity levels in a study involving adults with type 2 diabetes..$^{22}$ The annual cost to increase activity by $10 \%$ for the automated telephone surveillance and nurse care management was estimated to be $€ 551$ per participant. In contrast, two studies by Elley et $a^{11,27}$ used a threshold of $5 \times 30$ minutes of activity per week and found the cost per person to be $€ 57$ when predominantly doctor delivered, ${ }^{11}$ and $€ 331$ when nurse delivered. ${ }^{27}$ Most studies followed up participants for only 6-12 months, with only two costeffectiveness studies undertaken on the basis of a 2year trial. ${ }^{24,27}$

\section{Strengths and limitations of the study}

A strength of the current review is the inclusion of cost-effectiveness analyses that were based on randomised controlled trials. Accordingly, the quality of evidence included is high. However, estimates of cost-effectiveness are likely to be conservative because not all long-term benefits are accounted for in short-term randomised controlled trials. Modelled economic analyses suggest the cost-effectiveness of physical activity interventions may be even more favourable when long time horizons are taken into account. $^{33}$ The variability of outcome measures, interventions, target population groups, costs measured, and health-system variations in cost makes comparison of these cost-effectiveness studies difficult. With different infrastructures, funding models, and cost structures, it is also difficult to make comparisons between different countries. Furthermore, some studies included funder costs only, ${ }^{17,21-26}$ while others presented societal perspectives that also included costs to the participant. ${ }^{11,18-20,27}$ Consequently, there was a wide variation in costs per QALY gained between the studies. For example, the study of Gusi et al, involving a walking programme for older women who were overweight or had moderate depression, had the lowest cost per QALY of $€ 348 .{ }^{21}$ By comparison, the intervention with the highest cost per QALY of $€ 86877$ was an instructor-led walking programme (10 weeks, 2-3 times per week) for physically inactive 40-74-year-old adults. ${ }^{26}$ Even so, all interventions had cost-utility (cost per QALY) values within the range of pharmaceutical interventions that are currently considered for funding by governmental funding agencies. 


\section{Comparison with existing literature}

A previously published systematic review included eight studies involving interventions promoting physical activity, ${ }^{13}$ but included workplace and environmental interventions and economic modelling rather than actual costs. The previous review found that interventions directly targeting individual behaviour were able to promote the recommended levels of physical activity at a cost of about $€ 800$ per participant shifted to an active category over a 12month period. Interventions within general practices had the most favourable cost-effectiveness ratios (€106 per participant to reach at least $3300 \mathrm{~kJ}$ expended per week); however, the study was based on modelled rather than actual costs. ${ }^{34}$

Gordon et al reviewed the cost-effectiveness of a number of lifestyle interventions for smoking cessation, physical activity, diet, and alcohol reduction. ${ }^{35}$ The findings for physical activity interventions generally indicated favourable costeffectiveness: less than $€ 55860$ per QALY, and two studies showed net cost savings. ${ }^{36,37}$ Using a lifetime cost-effectiveness analysis from a societal perspective on a simulated cohort, Roux et al examined seven types of community-based physical activity interventions. ${ }^{33}$ All of the interventions evaluated were found to be cost-effective, with costs per QALY gained ranging from $€ 9763$ to $€ 46853$.

\section{Implications for clinical practice}

Due to the variability in study design and differences in outcome variables between the studies reviewed, it is difficult to draw firm conclusions about which types of interventions are most cost-effective. However, it appears that interventions such as 'exercise on prescription' delivered by primary care doctors or nurses, or brief advice delivered by mail-out or telephone are more cost-effective than intensive gym-based or instructor-led interventions. Furthermore, group exercise programmes appear to be more cost-effective than instructor-led walking programmes. Interventions delivered by nurses may be more cost-effective than when delivered by GPs.

Based on the higher-quality studies, it is possible to deliver a physical activity intervention for between $€ 1120$ and $€ 15860$ per QALY gained, which is more cost-effective than many other currently-funded pharmaceutical interventions. Therefore, physical activity interventions delivered in primary health care should be considered for funding at similar levels to currently-funded pharmaceutical interventions.

\section{Funding body}

The systematic review was funded by Sport and Recreation New Zealand (SPARC).

Ethics committee

Ethical approval was not required.

\section{Competing interests}

The authors have stated that there are none.

\section{Discuss this article}

Contribute and read comments about this article on the Discussion Forum: http://www.rcgp.org.uk/bjgp-discuss

\section{REFERENCES}

1. Oldridge NB. Economic burden of physical inactivity: healthcare costs associated with cardiovascular disease. Eur J Cardiovasc Prev Rehabil 2008; 15(2): 130-139.

2. Kahn EB, Ramsey LT, Brownson RC, et al. The effectiveness of interventions to increase physical activity. A systematic review. Am J Prev Med 2002; 22 (4 suppl): 73-107.

3. US Preventive Services Task Force. Behavioral counseling in primary care to promote physical activity: recommendation and rationale. Ann Intern Med 2002; 137(3): 205-207.

4. Stevens W, Hillsdon M, Thorogood M, McArdle D. Cost-effectiveness of a primary care based physical activity intervention in 45-74 year old men and women: a randomised controlled trial. Br J Sports Med 1998; 32(3): 236-241

5. Ministry of Health. A portrait of health: key results of the 2006/07 New Zealand Health Survey. Wellington: Ministry of Health, 2008.

6. Elley CR, Kerse N, Arroll B, Robinson E. Effectiveness of counselling patients on physical activity in general practice: cluster randomised controlled trial. BMJ 2003; 326(7393): 793.

7. Swinburn BL, Walter B, Arroll B, Russell D. The Green Prescription study: a randomized controlled trial of written exercise advice provided by general practitioners. Am J Public Health 1998; 88(2): 288-291.

8. Lawton BA, Rose SB, Elley CR, et al. Exercise on prescription for women aged 40-70 recruited through primary care: two year randomised controlled trial. BMJ 2008; 337: a2509.

9. Sørensen JB, Skovgaard T, Puggaard L. Exercise on prescription in general practice: a systematic review. Scand J Prim Health Care 2006; 24(2): 69-74.

10. Dalziel K, Segal L, Elley CR. Cost utility analysis of physical activity counselling in general practice. Aust NZ J Public Health 2006; 30(1): $57-63$.

11. Elley R, Kerse N, Arroll B, et al. Cost-effectiveness of physical activity counselling in general practice. N Z Med J 2004; 117(1207): U1216.

12. Elley C. The effectiveness and cost-effectiveness of the Green Prescription physical activity intervention: a cluster randomised controlled trial in primary health care. Auckland: University of Auckland, 2003. http://researchspace.auckland.ac.nz/handle/2292/3095 (accessed 27 Jan 2011).

13. Muller-Riemenschneider F, Reinhold T, Willich S. Cost-effectiveness of interventions promoting physical activity. Br J Sports Med 2009; 43(1): 70-76.

14. Drummond MF, O’Brien B, Stoddart GL, Torrance GW. Methods for the economic evaluation of health care programmes. 3rd edn. Oxford: Oxford University Press, 2005.

15. OANDA. Historical exchange rates. http://www.oanda.com/convert/fxhistory (accessed 27 Jan 2011).

16. Statistics New Zealand. Consumers Price Index: June 2008 quarter. http://www.stats.govt.nz/browse_for_stats/economic_indicators/CPI_i nflation/ConsumersPriceIndex_HOTPJun08qtraspx (accessed 27 Jan 2011).

17. UK Beam Trial Team. United Kingdom back pain exercise and manipulation (UK BEAM) randomised trial: cost effectiveness of physical treatments for back pain in primary care. BMJ 2004; 329(7479): 1381.

18. Cochrane T, Davey R, Matthes Edwards S. Randomised controlled trial of the cost-effectiveness of water-based therapy for lower limb osteoarthritis. Health Technol Assess 2005; 9(31): iii-iv, ix-xi, 1-114.

19. Hurley MV, Walsh NE, Mitchell HL, et al. Economic evaluation of a rehabilitation program integrating exercise, self-management, and active coping strategies for chronic knee pain. Arthritis Rheum 2007; 57(7): 1220-1229.

20. Hollinghurst S, Sharp D, Ballard K, et al. Randomised controlled tria of Alexander technique lessons, exercise, and massage (ATEAM) for chronic and recurrent back pain: economic evaluation. BMJ 2008; 337: a2656.

21. Gusi N, Reyes MC, Gonzalez-Guerrero JL, et al. Cost-utility of a walking programme for moderately depressed, obese, or overweight elderly women in primary care: a randomised controlled trial. $B M C$ 
Public Health 2008; 8: 231.

22. Handley MA, Shumway M, Schillinger D. Cost-effectiveness of automated telephone self-management support with nurse care management among patients with diabetes. Ann Fam Med 2008; 6(6): 512-518.

23. Sevick MA, Napolitano MA, Papandonatos GD, et al. Costeffectiveness of alternative approaches for motivating activity in sedentary adults: results of Project STRIDE. Prev Med 2007; 45(1) 54-61.

24. Munro JF, Nicholl JP, Brazier JE, et al. Cost effectiveness of a community based exercise programme in over 65 year olds: cluster randomised trial. J Epidemiol Commun Health 2004; 58(12): 1004-1010.

25. Dzator JA, Hendrie D, Burke V, et al. A randomized trial of interactive group sessions achieved greater improvements in nutrition and physical activity at a tiny increase in cost. J Clin Epidemiol 2004; 57(6): 610-619.

26. Isaacs A, Critchley J, Tai SS, et al. Exercise Evaluation Randomised Trial (EXERT): a randomised trial comparing GP referral for leisure centre-based exercise, community-based walking and advice only. Health Technol Assess 2007; 11(10): 1-165, iii-iv.

27. Elley CR, Garrett SM, Rose SB, et al. Cost-effectiveness of exercise on prescription with telephone support among women in general practice over 2-years. Br J Sports Med 2010; doi:10.1136/bjsm.2010.072439.

28. National Institute for Health and Clinical Excellence. Guide to the methods of technology appraisal. London: National Institute for Health and Clinical Excelllence, 2008.

http://www.nice.org.uk/media/B52/A7/TAMethodsGuideUpdatedJun e2008.pdf (accessed 27 Jan 2011).

29. Zhang P, Engelgau MM, Norris SL, et al. Application of economic analysis to diabetes and diabetes care. Ann Intern Med 2004; 140(11): 972-977.

30. Gilmer TP, Roze S, Valentine WJ, et al. Cost-effectiveness of diabetes case management for low-income populations. Health Serv Res 2007; 42(5): 1943-1959.

31. Cohen JT, Neumann PJ, Weinstein MC. Does preventive care save money? Health economics and the presidential candidates. $N$ Engl J Med 2008; 358(7): 661-663.

32. CDC Diabetes Cost-effectiveness Group. Cost-effectiveness of intensive glycemic control, intensified hypertension control, and serum cholesterol level reduction for type 2 diabetes. JAMA 2002; 287(19): 2542-2451.

33. Roux L, Pratt M, Tengs TO, et al. Cost effectiveness of communitybased physical activity interventions. Am J Prev Med 2008; 35(6): 578-588.

34. Sims J, Huang N, Pietsch J, Naccarella L. The Victorian Active Script Programme: promising signs for general practitioners, population health, and the promotion of physical activity. Br J Sports Med 2004; 38(1): $19-25$.

35. Gordon L, Graves N, Hawkes A, Eakin E. A review of the costeffectiveness of face-to-face behavioural interventions for smoking physical activity, diet and alcohol. Chronic Illn 2007; 3(2): 101-129.

36. Yu CM, Lau CP, Chau J. A short course of cardiac rehabilitation program is highly cost effective in improving long-term quality of life in patients with recent myocardial infarction or percutaneous coronary intervention. Arch Phys Med Rehabil 2004; 85(12): 1915-1922.

37. Treesak C, Kasemsup V, Treat-Jacobson D, et al. Cost-effectiveness of exercise training to improve claudication symptoms in patients with peripheral arterial disease. Vasc Med 2004; 9(4): 279-285. 